\title{
Exteroceptive vibration-induced finger flexion reflex in man
}

\author{
G ÖR A N E K L U N D, K A R L-ER I K H A G B A R T H, \\ A N D E R I K T O R E B J Ö R K
}

From the Department of Clinical Neurophysiology, Academic Hospital, Uppsala, Sweden

SUMMARY A tonic finger flexion reflex, induced by mechanical vibration $(100 \mathrm{~Hz})$ of the fingers, was studied in 20 healthy subjects. The outcome of nerve blocking experiments indicated that the reflex is mainly exteroceptive, distinct from the tonic vibration reflex of muscle spindle origin. Mechanoreceptors with fast conducting nerve fibres in the distal phalanx seem to constitute the major afferent source. We suggest that afferent signals from such receptors by a feedback mechanism can influence the motor performance of the exploring fingers.

It is an old observation that when the hand grasps a vibrating object, the voluntary finger flexion tends to become stronger than intended, and difficulties may be experienced in attempts to loosen the grip (Rood, 1860). The nature of this vibrationinduced grasping has been investigated in the present study. In particular, attempts were made to elucidate whether it is a proprioceptive tonic vibration reflex resulting from spread of the vibratory stimulus to muscle spindles in the finger flexor muscles (Hagbarth, 1973; Lance et al., 1973) or an exteroceptive reflex depending upon vibration-induced excitation of digital cutaneous mechanoreceptors. A preliminary report of the present study was presented at a recent symposium (Torebjörk et al., 1978).

\section{Methods}

The experimental subjects were 20 healthy volunteers of both sexes, aged 25 to 53 years. The two types of mechanical arrangements used to measure finger flexion or grasp force during vibration are illustrated in Fig. $1 \mathrm{~A}$ and $\mathrm{B}$. In the type $\mathrm{A}$ tests a small cylindrical DC motor $(15 \times 40 \mathrm{~mm})$ with an eccentric load on the axis was firmly attached to one of the fingers, usually on the volar side of the index finger. When the finger was unsupported the running motor produced finger vibrations which, measured at the finger tip, had an amplitude of

Address for reprint requests: Dr Karl-Erik Hagbarth, Department of Clinical Neurophysiology, Academic Hospital, 75014 Uppsala, Sweden. Accepted 20 December 1977
$0.5-1 \mathrm{~mm}$. The frequency of vibration could be varied from 50 to $200 \mathrm{~Hz}$. During the tests the hand was fixed to a support holding the wrist and fingers extended and the thumb abducted. The

A

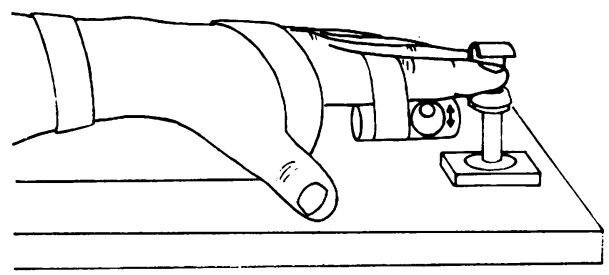

B

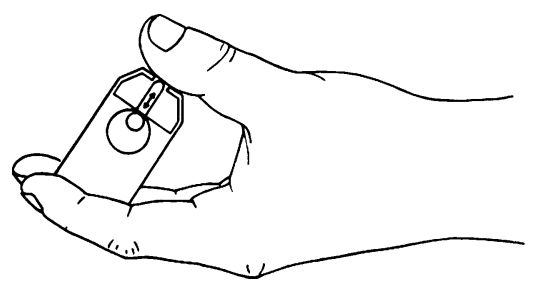

Fig. 1 Schematic presentation of the two types of experimental arrangements. A: the arrangements for most of the experiments. $B$ : for testing the effects of chlorethyl cooling. The double arrows indicate the main vibrating movements which in $A$ are transmitted to the index finger which in turn "oscillates" against the rigid force-transducer. In B the vibrating rod acts on the thumb pulp whereas the rest of the motor (only partly shown) is relatively still. The forcetransducer is in the frame on which the thumb is placed. Test situation $A$ is used in Figs. 2-7A and test situation B in Fig. $7 B$. 
pulp of the finger to which the vibrator was attached rested on a hard-rubber plate $\left(1 \mathrm{~cm}^{2}\right)$ connected to a strain gauge measuring finger flexion force. An upper plate positioned above the finger tip allowed measurements of finger extension force. The standard instruction given to the subjects was that they should maintain a weak constant effort in pressing the finger pulp against the plate. They were told not to counteract any force or positional change which they might perceive during the periods of vibration, which usually lasted for 30 seconds. In other tests the subjects were instructed either to remain completely passive and relaxed, or to exert maximal voluntary power in pressing the finger down against the supporting plate or up against the other plate on the dorsal side of the finger tip. Applications of the vibrator to other sites than indicated in Fig. 1A are described in the Results section.

In the type B tests the subject held the pulp of his thumb against a strain gauge plate with a hole in the centre through which a blunt motor-driven probe was moving up and down at a rate of $100 \mathrm{~Hz}$, causing skin indentations of about $0.5 \mathrm{~mm}$. Counter-pressure was exerted by the index finger, in this case not exposed to vibration. Also, in these tests, the instruction was to maintain a weak constant voluntary effort in holding the grip. The signals from the strain gauge were recorded on an X-Y recorder (Hewlett-Packard 7046A).

\section{Results}

REFLEX RESPONSE TO FINGER VIBRATION

On the standard instruction to maintain a weak finger flexion effort, all subjects responded to the index finger vibration with an involuntary increase in the flexion torque (Fig. 2B). There was usually an initial, relatively rapid rise in tension followed by a slower increment. With a vibration frequency of $100 \mathrm{~Hz}$ this increment often lasted for $20-30$ seconds and was succeeded by a relatively stable plateau level (Fig. 2C). The return to the control level at the end of the vibration period occurred within a few seconds. The height of the plateau level increased with increasing vibration frequencies up to about $130 \mathrm{~Hz}$. Frequencies higher than that produced little further enhancement but the level was reached more rapidly (Fig. 3). $100 \mathrm{~Hz}$ was chosen as the standard frequency in the experiments to be described.

On instruction to remain passive and relaxed, five of the subjects responded with a finger flexion reflex of similar time course but of lower strength than in the standard tests (Fig. 2A). In the remaining 15 subjects no measurable torque change occurred in the relaxed state.

The strength of the reflex, as measured in the standard tests, varied from one subject to the next but was relatively constant on repeated trials in the same subject. In subjects with strong reflexes the plateau level reached 4-5 N, corresponding to about one-fifth of their maximal voluntary finger flexion power in this particular position of the hand and fingers (Fig. 4A and $\mathrm{B}$ ).

Vibration applied while the subject was exerting maximal finger flexion power produced no consistent change in torque (Fig. 4B). By contrast, a reduction of extension torque was observed when vibration was applied while the subject was exerting maximal voluntary extension power (Fig. 4C). This reduction of the extension force was not studied further in the present investigation.
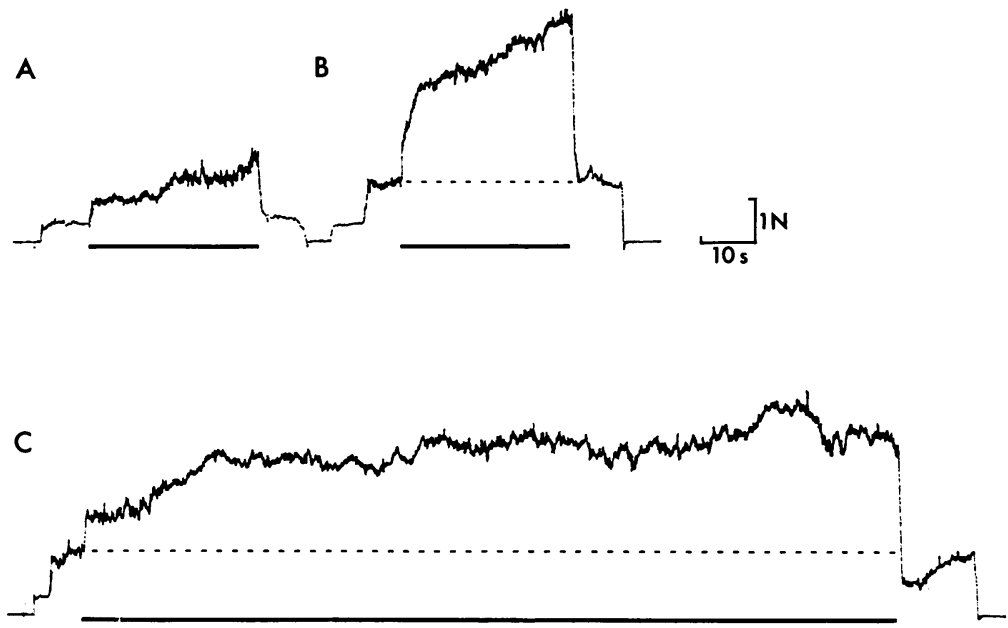

Fig. 2 Effect of voluntary background contraction $(B)$ and prolonged vibration (C). In $A$ the plateau of the force before and after vibration (bar) is due to the weight of the index finger plus the small vibrator; in $B$ the subject maintains $a$ weak constant flexion effort against the transducer as indicated by the hatched line. In $A$ and $B 30 s$ vibration (bar), in $C$ almost 2.5 min vibration. 


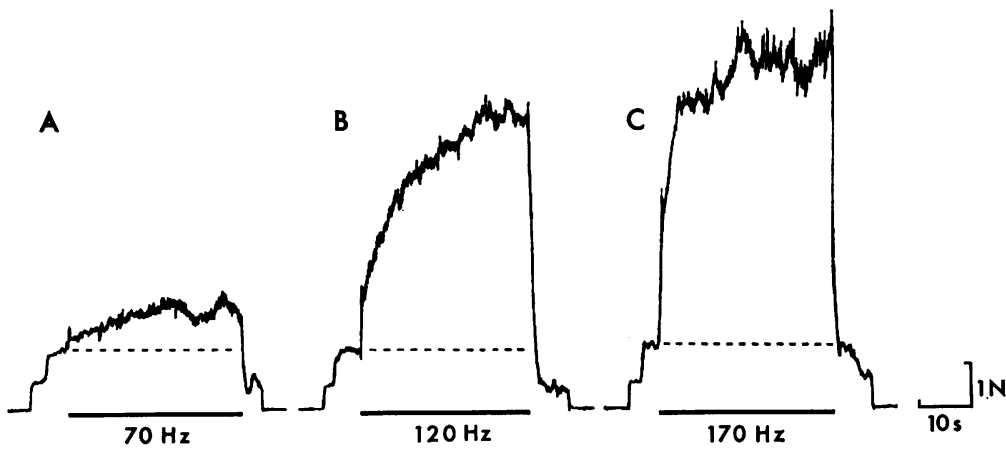

Fig. 3 Effect of different vibration frequencies. The subject maintains a weak voluntary flexion as in Fig. $2 B$ and $C$. In $A, B$, and $C$ the vibration frequencies are indicated below the vibration bars.

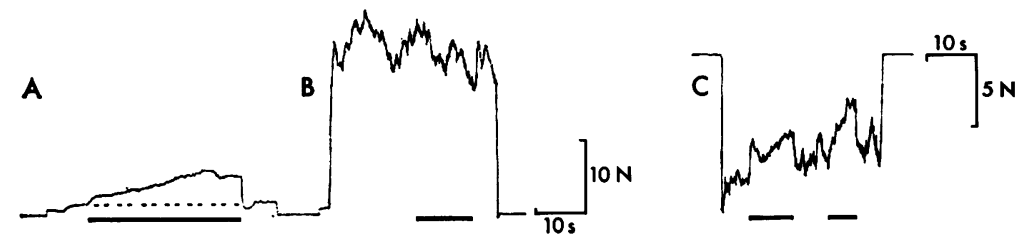

Fig. 4 Effects of vibration on the maximal ability to flex $(B)$ and to extend (C) the finger. A shows the strength of the reflex with the subject maintaining $a$ weak voluntary flexion. $B$ shows subject maintaining maximal voluntary flexion force with no apparent effect of vibration. $C$ shows maximal voluntary extension force (note different zero level and gain). The two periods of vibration cause a reduction of the force.

FINGER VIBRATION COMPARED WITH VIBRATION OVER THE FINGER FLEXOR MUSCLES

Without exception, a much stronger increase in finger flexion torque developed when the vibrator was attached to the volar side of the finger than when it was attached over the finger flexor muscles or their tendons. Furthermore, the weak tonic vibration reflex occurring in response to vibration over the flexor muscles did not show the very slow build-up typical of the motor response to finger vibration (Fig. 5).
VIBRATOR APPLIED TO THE DORSUM OF THE INDEX FINGER AND TO NEIGHBOURING FINGERS

Application of the vibrator to the dorsum of the index finger gave a similar but somewhat weaker motor response than when it was applied on the volar side. Application on the middle finger or the thumb (with the pulps resting on a support in a similar way as the index finger) had in some subjects no effect on the torque produced by the index finger; in others it produced a small increase in torque always much smaller than when the

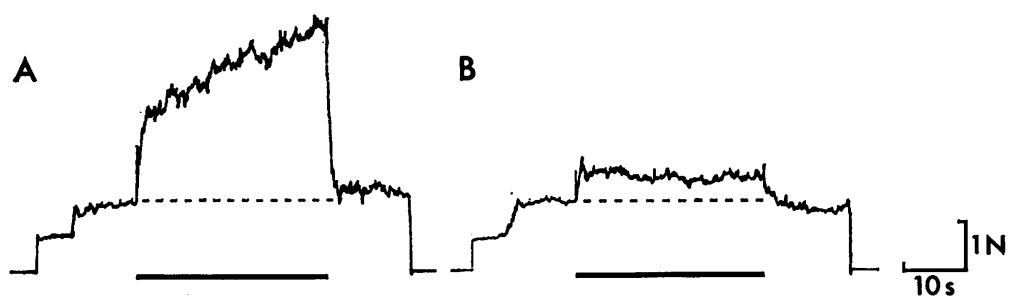

Fig. 5 Response to index finger vibration $(A)$ compared to response arising when vibration is applied over the finger flexion tendons, just proximal to the wrist $(B)$. 
index finger itself was vibrated (Fig. 6). Further tests on different fingers showed that the flexion torque increased in the finger exposed to the vibratory stimulus whereas torque exerted by neighbouring fingers was largely unaffected.

\section{PERCEPTIONS ACCOMPANYING THE FINGER VIBRATION} REFLEX

All subjects reported that in the standard tests they perceived a slow increase in finger flexion torque, not produced intentionally but occurring as a result of the vibration. Most of them also reported some type of illusory positional change during vibration-for example, an upward slow movement of the whole finger accompanied by flexion of the interphalangeal joints. When vibration suddenly stopped they had a feeling of "relaxation" as the illusory positional change gradually disappeared. At this stage some subjects had difficulties in following the instruction to maintain a constant voluntary motor effort, and in the post-vibratory period the torque sometimes stayed at a different level than before vibration. A frequent comment was that a feeling of numbness remained in the finger tip a few minutes after the vibration tests.

\section{EFFECT OF DIGITAL NERVE BLOCKS}

In one subject lidocaine $(1 \%)$ was injected around the base of the index finger in doses sufficient to cause total anaesthesia of the whole finger. In another, the anaesthesia was restricted to the distal phalanx by injecting the anaesthetic around the distal interphalangeal joint (Fig. 7A). Similar results were obtained in these two experiments in which repeated vibration tests of the standard

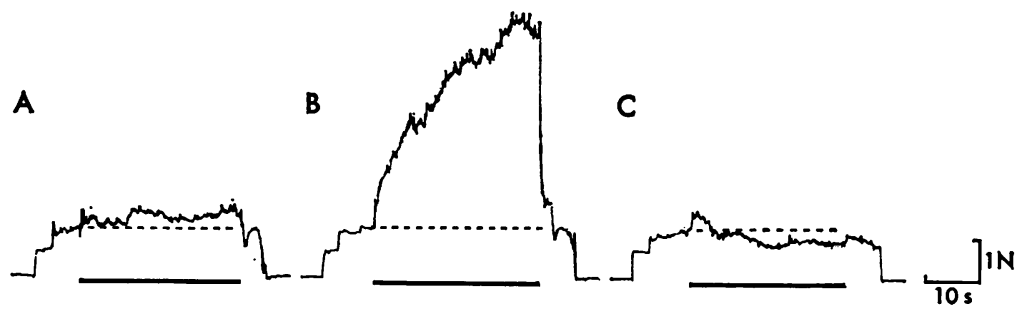

Fig. 6 Limited spread of the vibration-induced finger flexion reflex. The index finger flexion force was measured during vibration of the third finger $(A)$, the index finger $(B)$, and the thumb $(C)$. In $A$ and $C$ a passive support against the pulp of the vibrated finger was used to make the stimulus equally effective (see text).

A

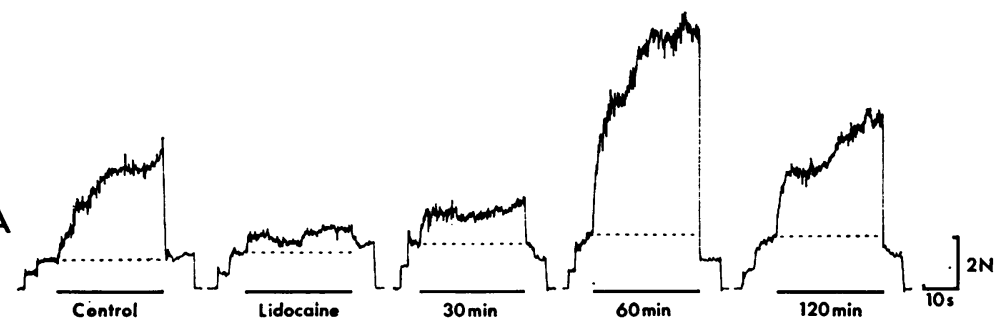

B

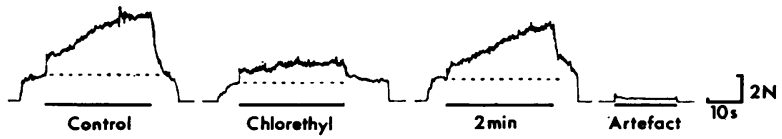

Fig. 7 Effect of local anaesthesia. A: lidocaine anaesthesia of the distal phalanx of the index finger. B: test situation $B$ (cf. Fig. 1B), localised cooling of a small skin area beneath the vibrating rod in the pulp of the thumb. Artefact denotes a small false "force level" with this particular vibrator. In both $A$ and $B$ the subjects maintained a weak voluntary flexion. Type of test, time after application, and calibration are marked. 
type (Fig. 1A) were performed before and during the anaesthesia, as well as during the recovery period lasting about two hours. While the anaesthesia was complete there was a marked reduction of the motor response to the vibratory stimulus, the remaining weak response being reminiscent of that produced by vibration over the finger flexor tendons at the wrist (cf. Fig. 5B). In the control sequences between vibrations, the subjects succeeded quite well in maintaining a constant weak flexion torque, even though they did not perceive the finger pulp contact with the plate. The running vibrator produced no sensations from the finger pulp nor any sensations of increasing torque. The subject with the whole finger anaesthetised reported that he was unable to perceive the vibratory stimulus.

The first signs of a recovering motor response to vibration appeared at about the same time as the subjects started to report returning vibration sensibility. Then came a period lasting about 15 minutes when in both subjects the motor response was enhanced as compared with the controls before the anaesthesia (Fig. 7A). One of the subjects reported that, during this period, he had paraesthesias in the finger pulp with marked tingling sensations arising during vibration and also in response to local taps on the finger tip. Both subjects were, at this stage, still unable to perceive pain and temperature changes in the finger tip. The motor response returned to its control level at about the same time as the subjects regained normal vibration sensibility.

\section{EFFECT OF CHLORETHYL ANAESTHESIA OF THE FINGER PULP}

In the type $B$ tests (Fig. 1B), the changes in grasp force induced by vibration on the pulp of the thumb were measured before and immediately after the pulp skin had been cooled by chlorethyl spray. In the control runs before the anaesthesia the vibration produced a slow involuntary increase in grasp force reaching about $4 \mathrm{~N}$ (Fig. 7B). During the pulp anaesthesia immediately after the chlorethyl spray, such a gradual increase in torque did not occur. There remained, however, a weaker sustained motor response reaching a plateau at about $1 \mathrm{~N}$ (Fig. 7B). A response of this latter type could also be elicited by vibration over the thenar muscle.

\section{Discussion}

Clinical observations on patients with partial or total loss of finger sensibility from peripheral nerve lesions have shown that impulses from cutaneous receptors are very important for the motor control of the hand (Moberg, 1972). It is also well-known that a grasp reflex can be elicited in newborn babies by weak tactile stimuli in the palm of the hand or on the fingers. A similar reflex, probably originating mainly from exteroceptors in the glabrous skin (Shahani et al., 1970) becomes overt in the adult only under pathological conditions, the "forced grasping," being indicative of a central nervous disorder usually involving frontal lobe lesions. Possibly the vibration reflex analysed in the present study may be regarded as an artificially induced grasp reflex which becomes overt in healthy adults by the intense vibration-induced stimulation of tactile end organs.

The results of the nerve blocking experiments indicate that the finger vibration reflex is mainly exteroceptive, distinct from the tonic vibration reflex of muscle spindle origin. Mechanoreceptors in the distal phalanx (vibrating against the supporting plate in the type A tests) seem to constitute the major afferent source. These receptors are probably supplied with fast conducting fibres, since the reflex could be elicited when the subjects were unable to perceive temperature changes and pain, indicating block of impulse transmission in slowly conducting $\mathrm{A}$ and $\mathrm{C}$ fibres (Hallin and Torebjörk, 1976). As judged by the effect of the chlorethyl anaesthesia, the end organs mainly responsible are probably located rather superficially in the glabrous skin. Recent work by Johansson and Vallbo (1976) has shown that rapidly adapting RA receptors and slowly adapting SA-I receptors are very numerous in the glabrous skin of the human finger tips. It is suggested that receptors of this type constitute the major afferent source of the vibration-induced flexion. During recovery from the nerve block these sensory units probably passed through a state of hyperexcitability, resulting in skin paraesthesias and an enhancement of the motor response.

With their high sensitivity to mechanical stimulation combined with small receptive fields, the RA receptors are likely to be important for the sensory discrimination of textures. The present results indicate that afferent signals from such receptors by a feedback mechanism can influence the motor performance of the exploring fingers. The findings are compatible with those obtained with a different technique by Marsden et al. (1977) who found that anaesthesia of the thumb may abolish the compensatory increase in active force normally arising when a voluntary thumb flexion movement is suddenly opposed by an external force (see also Gandevia and McCloskey, 1977). They also noted that it needed a greater 
effort on the part of the subject to overcome a resistance and move the thumb when it was anaesthetised. Their suggestion that long loop cortical reflex arcs are involved is not necessarily applicable for the tonic finger vibration reflex. Alternative possibilities would be that the mechanoreceptive impulses from the finger reinforce the descending activation through convergence either on the flexor motoneurones themselves or on spinal interneurones which mediate the command to the flexor muscles (Lundberg, 1972). The gradual increase of the vibration reflex suggests that some type of slowly augmenting central facilitatory process is initiated and maintained by the vibration-induced afferent barrage. Since such longlasting, slowly adapting processes may operate at the spinal level (Hultborn and Wigström, 1978) the gradual increase of the response does not in itself exclude a spinal reflex arc. Evidence in favour of a segmental feedback regulation of the grip of the hand is not lacking. Thus, Stephens et al. (1976), using an averaging technique, found that mechanical taps on a finger tip or weak electrical shocks on a digital nerve elicited a reflex response of very short latency in the first dorsal interosseous muscle, a reflex which they considered to be exteroceptive since it was abolished by finger anaesthesia.

Handling of vibrating objects is common in different types of industrial work, and different types of neural and vascular disorders have been described as a result of long-term exposure to vibration (Hyvärinen et al., 1973). Less attention has been paid to the question of how in healthy subjects the precision in manipulatory movements is affected when the tools to be handled are vibrating. It also remains to be elucidated how finger vibration affects motor performance in patients with different types of peripheral and central neural disorders.

This study was supported by the Swedish Medical Research Council (Project No. B78-14X-02881-09A).

\section{References}

Gandevia, S. C., and McCloskey, D. I. (1977). Effects of related sensory inputs on motor performances in man studied through changes in perceived heaviness. Journal of Physiology, 272, 653-672.
Hagbarth, K.-E. (1973). The effect of muscle vibration in normal man and in patients with motor disorders. In New Developments in Electromyography and Clinical Neurophysiology, Vol. 3, pp. 428-443. Edited by J. E. Desmedt. S. Karger: Basel.

Hallin, R. G., and Torebjörk, H. E. (1976). Studies on cutaneous $\mathrm{A}$ and $\mathrm{C}$ fibre afferents, skin nerve blocks and perception. In Sensory Function of the Skin in Primates, with Special Reference to Man, pp. 137-149. Edited by Y. Zotterman. Pergamon Press: New York.

Hultborn, H., and Wigström, W. (1978). Motor responses with long latency and maintained duration evoked by activity in Ia afferents. In Progress in Clinical Neurophysiology, Vol. 8. Edited by J. E. Desmedt. S. Karger: Basel. In press.

Hyvärinen, J., Pyykkö, I., and Sundberg, S. (1973). Vibration frequencies and amplitudes in the aetiology of traumatic vasospastic disease. Lancet, 1, 791-794.

Johansson, R., and Vallbo, A. B. (1976). Skin mechanoreceptors in the human hand. An inference of some population properties. In Sensory Function of the Skin in Primates, with Special Reference to Man, pp. 171-184. Edited by Y. Zotterman. Pergamon Press: New York.

Lance, J. W., Burke, D., and Andrews, C. J. (1973). The reflex effects of muscle vibration. In New Developments in Electromyography and Clinical Neurophysiology, Vol. 3, pp. 444-462. Edited by J. E. Desmedt. S. Karger: Basel.

Lundberg, A. (1972). The significance of segmental spinal mechanisms in motor control. In Proceedings of the 4th International Biophysics Congress, Moscow.

Marsden, C. D., Merton, P. A., and Morton, H. B. (1977). The sensory mechanism of servo action in human muscle. Journal of Physiology, 265, 521535.

Moberg, E. (1972). Fingers were made before forks. Hand, 4, 201-206.

Rood, O. N. (1860). On contraction of the muscles induced by contact with bodies in vibration. American Journal of Science and Arts, 24, 449.

Shahani, B., Burrows, P., and Whitty, C. W. M. (1970). The grasp reflex and preservation. Brain, 93, 181-192.

Stephens, J. A., Usherwood, T. P., and Garnett, R. (1976). Technique for studying synaptic connections of single motoneurones in man. Nature, 263, 343.

Torebjörk, H. E., Hagbarth, K.-E., and Eklund, G. (1978). Tonic finger flexion reflex induced by vibratory activation of digital mechanoreceptors. In Active Touch. Edited by G. Gordon. Pergamon Press: New York. In press. 